\title{
Research of the shell vibration and radiated noise based on finite element method
}

\author{
Yuting Sun ${ }^{1, a}$, Juan $\mathrm{Xu}^{1, \mathrm{~b}}$ and Tong Liu ${ }^{2}$ \\ ${ }^{1}$ Shandong Provincial Key Laboratory of Ocean Environment Monitoring Technology, Institute of \\ Oceanographic Instrumentation, Shandong Academy of Sciences, Zhejiang Road 28, Qingdao \\ 266001, China \\ ${ }^{2}$ Qingdao University of Science and Technology \\ asunyuting_sdioi@163.com, bxujuan101066@163.com
}

Keywords: shell vibration; finite element; modal analysis; radiated noise

\begin{abstract}
The electromotor shell is analyzed by the finite element method. The result shows that vibrations of exterior and the top of the shell produce the shell vibration. The rib reinforcement type and the surrounded structure are designed to optimize and ameliorate the prototype structure. Then vibration and noise are analyzed. From the results it can be seen that amplitude at the top in the rib reinforcement type is suppressed effectively, while amplitude in the exterior is large. The surrounded layer in the surrounded structure successfully breaks the bridge of the inner cylinder to the outer cylinder. Then the vibration of electromotor is absorbed by the surrounded layer and the vibration reduces obviously. The acoustic field mean of the surrounded layer is lowest in the three structures, which effectively reduces the radiated noise from the top of the electromotor shell.
\end{abstract}

\section{Introduction}

The shell is the main noise source of the electromotor radiation. So researching the vibration noise is the imminence requirement for reducing the electromotor noise [1-2]. In this paper the characteristic of the shell structure is analyzed and improved. The structure is improved and optimized combined with the modal analysis and noise analysis. At the same time methods of reducing vibration and noise in shell parts are discussed and analyzed, which provides a base for controlling vibration and noise.

\section{The finite element method of structure modal analysis}

The acoustic modal analysis method is used to obtain the vibration noise under the work condition of the shell. Generally the acoustic modal analysis method is used to solve the zero of the generalized force vector in the acoustic wave equation. That is the acoustic resonance frequency and the distribution of sound pressure when the motion vector of boundary structure meets the equation of $U_{e}=\dot{U}_{e}=\ddot{U}_{e}=0$. Then different boundary conditions are applied to the model established by the finite element model to analyze the corresponding acoustic modal analysis.

For the high-reflective surface with a material that does not "absorb" the sound, the equation $U_{e}=\dot{U}_{e}=\ddot{U}_{e}=0$ is substituted in the equation to obtain the differential equation under the boundary condition.

$$
\left[M_{e}^{p}\right]\left\{\ddot{p}_{e}\right\}+\left[K_{e}^{p}\right]\left\{p_{e}\right\}=\{0\}
$$

The characteristic equation is as follows.

$$
\left[\left\{K_{e}^{P}\right\}-\omega^{2}\left\{M_{e}^{P}\right\}\right]\left\{P_{e}\right\}=0
$$


For the high-reflective surface with a sound absorbing material, the equation of $U_{e}=\dot{U}_{e}=\ddot{U}_{e}=0$ is substituted in the attenuation equation to obtain the differential equation under the boundary condition.

$$
\left[M_{e}^{P}\right]\left\{\ddot{P}_{e}\right\}+\left[C_{e}^{P}\right]\left\{\dot{P}_{e}\right\}+\left[K_{e}^{P}\right]\left\{P_{e}\right\}=\{0\}
$$

The characteristic equation is as follows.

$$
\left[\left\{K_{e}^{P}\right\}+i \omega\left\{C_{e}^{P}\right\}-\omega^{2}\left\{M_{e}^{P}\right\}\right]\left\{P_{e}\right\}=0
$$

Then equations are calculated by the numerical simulation method to obtain the acoustic resonance frequency $\omega_{j}(j=1,2,3 \cdots, n)$ and the distribution of sound pressure $P_{j}(j=1,2,3 \cdots, n)$ with the acoustic model under different boundary conditions of the shell cavity. When all structural constraints are applied to the boundary condition, the wall in the acoustic modal is rigid. While when structural constraints are not applied to the boundary condition, the siding and the cavity of the shell freely vibrate.

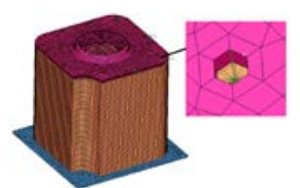

Fig 1 Calculation model and mesh

\section{Structural model and mesh}

The motor shell is made up of the sidings. With the establishment of the finite element model of the shell's external surface, that the shell connection is rigid is supposed and the flexing action of rubber airproof strip is not considered. The shell model is shown in Fig.1. The shell is simplified as the plate with uniform thickness. Because 3D solid element model influences calculation precision, 2D shell element is used for the mesh. The connection positions of the bolts are restricted for full freedoms of the bottom. The bolts' positions with three layers of the lid on the top are connected with the RBE2 unit, as shown in Fig.1. The material of the motor shell is ABS, elasticity modulus is 3.0E9Pa, poisson's ration is 0.25 and the density is $2500 \mathrm{~kg} / \mathrm{m}^{\wedge} 3$.

\section{Vibration analysis}

The overlap of the input pumping frequency of the rotor and some natural frequency of the shell will form the structure resonance, which cause the obvious vibration generated. Then the noise is produced. The shell is analyzed with modal. The excitation force $1000 \mathrm{~Pa}$ is applied to the contact parts of the electromotor with the shell, with the excited frequency from $20 \mathrm{~Hz}$ to $1000 \mathrm{~Hz}$. Then each order formation and natural frequency are obtained. At the same time vibration performance is mastered. The fluid media in the cavity of the shell is air. Without considering the coupling, the result of model analysis is obtained by the finite element method in Fig.2. By comparative analysis of the vibratory response charts from $100 \mathrm{~Hz}$ to $1000 \mathrm{~Hz}$, amplitude in the exterior increases with the frequency. The amplitudes at the top and the bottom of the shell increase gradually with the increasing frequency. This shows that the shell vibration is mainly from vibrations of exterior and the top of the shell. 


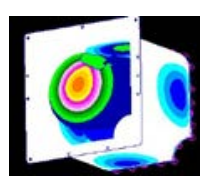

(a) $100 \mathrm{~Hz}$

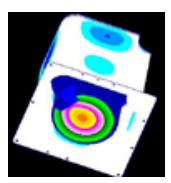

(f) $100 \mathrm{~Hz}$

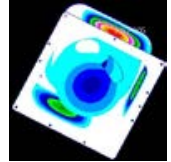

(b) $100 \mathrm{~Hz}$



(g) $100 \mathrm{~Hz}$

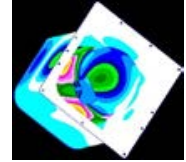

(c) $100 \mathrm{~Hz}$



(h) $100 \mathrm{~Hz}$

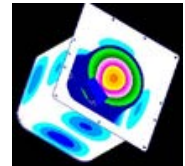

(d) $100 \mathrm{~Hz}$

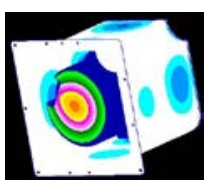

(i) $100 \mathrm{~Hz}$

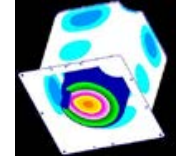

(e) $100 \mathrm{~Hz}$

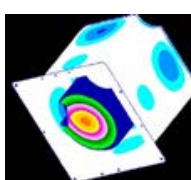

(j) $100 \mathrm{~Hz}$

Fig 2 The vibratory response charts

\section{Structure optimization and noise analysis}

The amplitude in the surrounded and the top of the shell is large after excited. The structure can be changed by adding the bracing structure in interior to reducing the vibration. The radiated noise from the electromotor driving can be reduced by pasting the absorbing material in the internal surface and external surface. The surrounded structure is used to control the overall noise. As shown in Fig.4, two improved structures are designed combined with the acoustic packet technology to improve the shell strength and reduce the vibration. The rib reinforcement structure is used in Fig.3 (a), which connects the inner barrel and the outer barrel. The vibration attenuation layer is set between the inner barrel and the outer barrel in Fig.3 (b). The vibration and noise of the two above structures are analyzed by the finite element method.

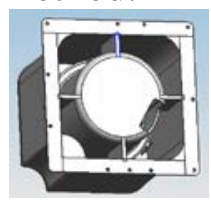

(a)The rib reinforcement type

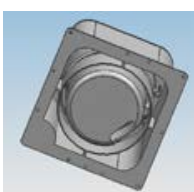

(b)The surrounded structure type

Fig 3 Chart of structural optimization and improvement

Vibration charts of the two improved structures in different frequencies are shown in Fig. 4 and Fig.5. By comparative analysis, it can be found that amplitude of the surrounding wall of the shell increases obviously. This is because the inner barrel is connected with the outer barrel, and the vibration is transmitted from the inner barrel and the rib reinforcement to the exterior. Then amplitude of the top is effectively inhibited. From the Fig.5, it can be found that the vibrations of the surrounding wall and the top are effectively reduced with the surrounded structure. It is mainly because the surrounded layer in the surrounded structure successfully breaks the bridge of the inner cylinder to the outer cylinder. Then the vibration of electromotor is absorbed by the surrounded layer and the vibration reduces obviously.

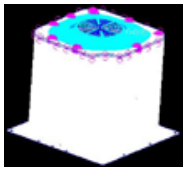

(a) $100 \mathrm{~Hz}$

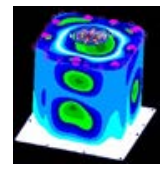



(b) $200 \mathrm{~Hz}$

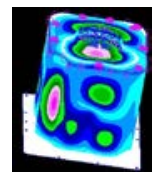

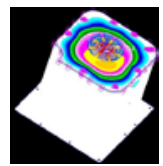

(c) $300 \mathrm{~Hz}$



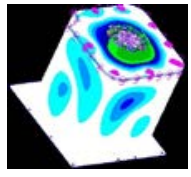

(d) $400 \mathrm{~Hz}$



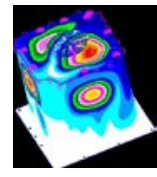

(e) $500 \mathrm{~Hz}$

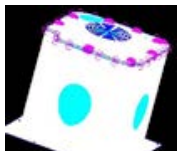

Fig 4 Vibration response chart with the rib reinforcement type 


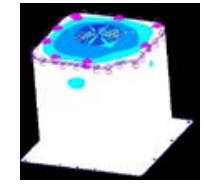

(a) $100 \mathrm{~Hz}$

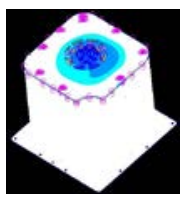

(f) $600 \mathrm{~Hz}$

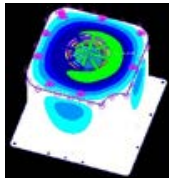

(b) $200 \mathrm{~Hz}$

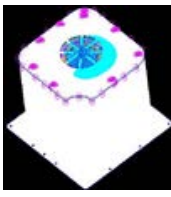

(g) $700 \mathrm{~Hz}$

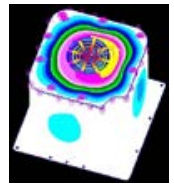

(c) $300 \mathrm{~Hz}$

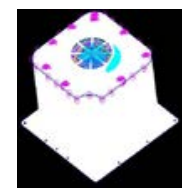

(h) $800 \mathrm{~Hz}$



(d) $400 \mathrm{~Hz}$

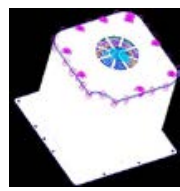

(i) $900 \mathrm{~Hz}$



(e) $500 \mathrm{~Hz}$

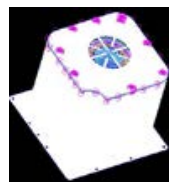

(j) $1000 \mathrm{~Hz}$

Fig 5 Vibration response chart with the surrounded structure type

It is found that vibration can be effectively reduced with the surrounded structure. The radiated noises with three different structures are analyzed by the finite element method. And the radiated noise charts are shown in Fig.6 and Fig.7. The noise source of the prototype structure is on the top of the shell, while noise source of the rib reinforcement type is from the surrounding wall, as the result of structure vibration distribution. The acoustic field mean of the surrounded layer is lowest in $200 \mathrm{~Hz}$, which effectively reduces the radiated noise from the top of the electromotor shell.
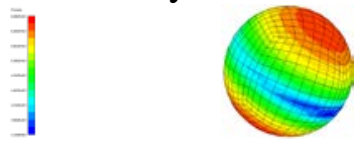

(a)the prototype
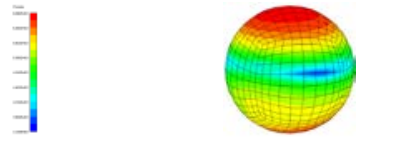

(a)the prototype

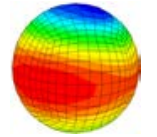

(b)The rib reinforcement type

Fig 6 The acoustic field in $200 \mathrm{~Hz}$



(c) The surrounded type

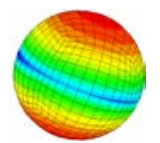

(b)The rib reinforcement type

Fig 7 The acoustic field in $1000 \mathrm{~Hz}$

\section{Conclusions}

The electromotor shell is analyzed by the finite element method. The result shows that vibrations of exterior and the top of the shell produce the shell vibration. The rib reinforcement type and the surrounded structure are designed to optimize and ameliorate the prototype structure. Then vibration and noise are analyzed. From the results it can be seen that amplitude at the top in the rib reinforcement type is suppressed effectively, while amplitude in the exterior is large. The surrounded layer in the surrounded structure successfully breaks the bridge of the inner cylinder to the outer cylinder. Then the vibration of electromotor is absorbed by the surrounded layer and the vibration reduces obviously. The acoustic field mean of the surrounded layer is lowest in the three structures, which effectively reduces the radiated noise from the top of the electromotor shell.

\section{Acknowledgements}

This work was financially supported by the research foundation of Qingdao Science and Technology Development (13-1-4-245-jch) 


\section{References}

[1] W.B. Jeong, et al., Finite element vibration analysis of cylindrical shells conveying fluid with considering acoustic-structure interactions. Jsme International Journal Series C-Mechanical Systems Machine Elements and Manufacturing, Vol.49(2006): p. 488-493.

[2] S. Merz, et al., Development of coupled FE/BE models to investigate the structural and acoustic responses of a submerged vessel. Journal of Computational Acoustics, Vol. 15(2007) , p. 23-47. 